\title{
1 The switching between zonal and blocked mid-latitude atmospheric circulation: a dynamical system perspective
}

\author{
Davide Faranda · Giacomo Masato · Nicholas \\ Moloney · Yuzuru Sato • Francois Daviaud • \\ Bérengère Dubrulle • Pascal Yiou
}

Received: September 14, 2015/ Accepted:

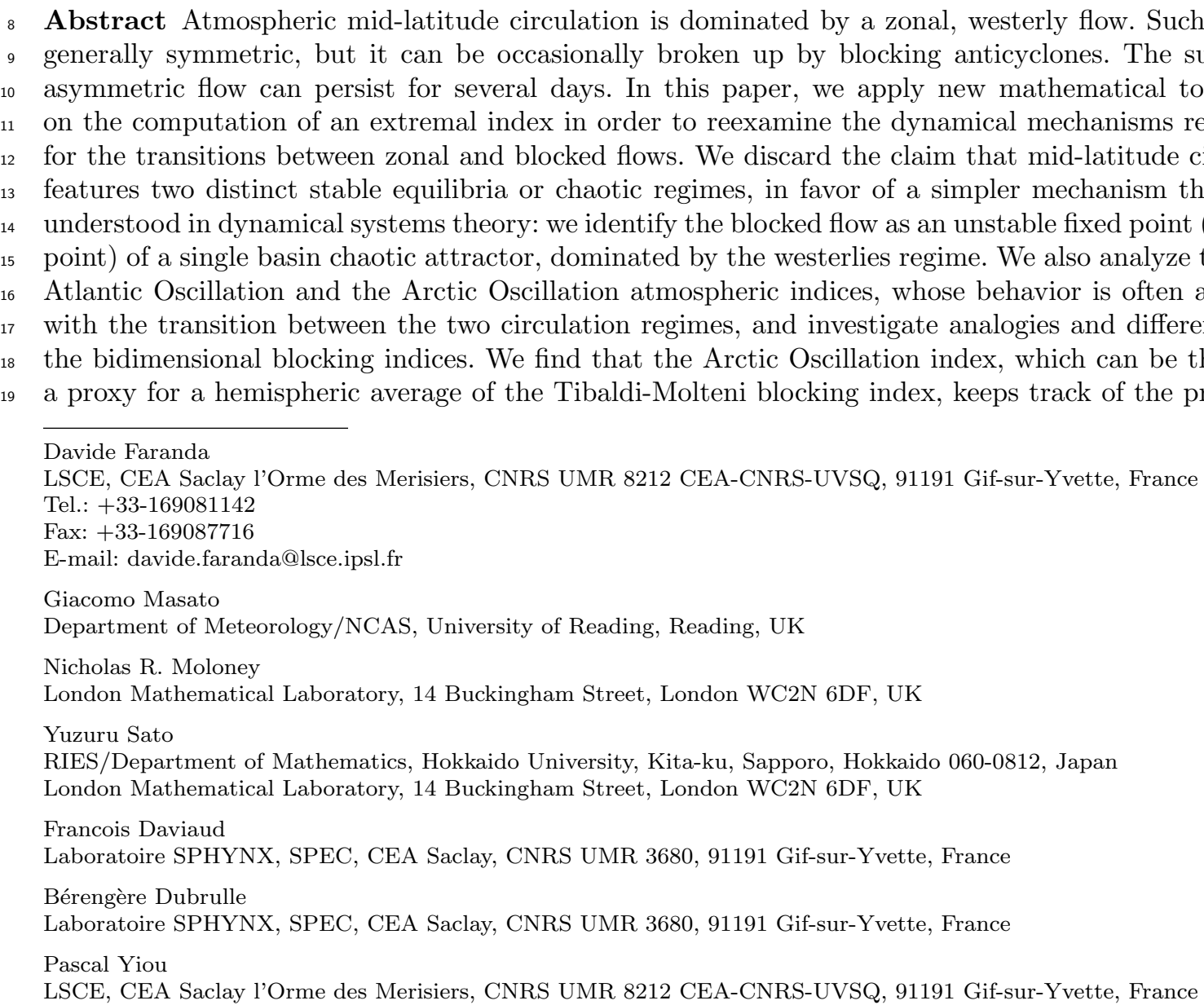


unstable fixed points. On the other hand, the North Atlantic Oscillation, representative only of local properties of the North Atlantic blocking dynamics, does not show any trace of the presence of unstable fixed points of the dynamics.

Keywords Blocking Index, Mid-latitude circulation, Extremal index

\section{Introduction}

In the time range of $2-8$ days, the mid-latitude large-scale circulation is mainly driven by the destabilization of a westerly sheared jet, associated with a meridional temperature gradient (Holton and Hakim 2013). The destabilizing mechanism is referred to as the baroclinic instability and it consists in the appearance of three dimensional wave structures (extra-tropical cyclones and anticyclones) normally embedded in the mid-latitude westerlies. The minimal model for such an instability is known as the Charney-Eady model (Charney 1947). Such a model is based on the stability analysis of the quasi-geostrophic potential vorticity equation coupled with a thermodynamic equation. The stability parameter is the Burger number, i.e. the ratio between stratification (a quantity linked to the meridional temperature gradient) and rotational effects.

Cyclones and anticyclones are generally embedded in the mid-latitude jet, and have average lifetimes of a few days that depend on their size, longitudinal asymmetry and interaction with the topography (Emanuel 2005; Rudeva 2008). However, a few times per year and with higher frequency in the winter season, large high-pressure structures may form and persist for several days, breaking up the westerlies circulation and forcing the jet to move towards higher latitudes or even split up into two branches, hence breaking the longitudinal symmetry. This kind of circulation is referred to as blocked flow (Charney and DeVore 1979). During blocking conditions a few extreme climate events have occurred like the December 2010 cold spell in northern and central Europe or the summer 2003 heatwave over western Europe (Schär and Jendritzky 2004; Beniston 2004) or the 2010 heatwave over Russia (Dole et al 2011; Barriopedro et al 2011). It is therefore crucial to get a deeper understanding of the blocked flow and of the mechanism which regulates the transition to the westerlies regime.

Besides the anticyclones embedded in the mid-latitude jet, there is a large fraction of subtropical anticylones such as the Azores high that are located further south and are quasi-stationary. Since it is necessary to discern between typical blocking highs in the mid to high latitudes and those at lower latitudes, in this paper, we will focus only on the blocking occurring between mid to high latitudes, deferring to another publication the study of lower latitudes for which other robust blocking detection algorithms have been devised (Barriopedro et al 2006, 2010; Lupo and Smith 1995; Scherrer et al 2006).

In order to understand the transition between those flow regimes, many studies of mid-latitude dynamics have been conducted both theoretically and experimentally. Legras and Ghil (1985) and Ghil (1987) have shown the intricacy of such circulation by studying an intermediate complexity model of a barotropic flow with dissipation forcing and topography. They observed two distinct equilibria which can be associated with either the westerlies or the blocked flows. Similar conclusions appear in (Mo and Ghil 1988) and are supported by experimental laboratory studies (Weeks et al 2000). A vast amount of literature points to very different mechanisms. Some of these theories relate blocking to resonant amplification of free quasistationary Rossby waves (Tung and Lindzen 1979) whereas others have considered barotropic (Simmons et al 1983) or baroclinic instability (Frederiksen 1982) as the main mechanism. The development of nonlinear theories has led some authors to regard blocking episodes as a manifestation of multiple equilibria 
in asymmetrically forced flows (Hansen 1986) or as a result of soliton-modon structures (McWilliams et al 1981). A significant number of works invoke non-linear interactions, either between zonal flow and eddies (Charney and DeVore 1979), or between planetary waves (Egger 1978; Kung et al 1990; Christensen and Wiin-Nielsen 1996). Other authors have analyzed the relationship between blocking anticyclones and lowfrequency planetary waves at the hemispheric scale (Wallace and Blackmon 1983; Dole 1986). A number of authors have pointed to the decisive contribution from high frequency cyclones to develop (Colucci and Alberta 1996; Nakamura et al 1997) and sustain (Shutts 1986) the typical low-frequency anomalies associated with blocking episodes. Conceptual models linking blocking anticyclones and transient eddies have been proposed (Shutts 1983). On the basis of observations some other works see blocking appearing as a distinctive dynamical feature with respect to westerlies circulation, but it consists of several multistable patterns (Vautard 1990; Masato et al 2009, 2012).

In this paper we reanalyse data over the past decades to detect whether the dynamics of blocking is compatible with the existence of an unstable fixed point of the atmospheric mid-latitude circulation. This evidence comes from dynamical systems theory and is supported by the common experience that, within the blocked flow, atmospheric variables follow a highly predictable dynamics (persistence of the same weather conditions for several days), whereas in the zonal flow they mostly have a chaotic behavior (irregular alternation between cyclonic and anticyclonic phases). Such kind of dynamical features are also encountered for several dynamical systems systems ranging from toy models as the Pomeau-Manneville map, the Hénon map or the Lorenz equations (Benzi et al 1985) to intermediate complexity models (Payne and Sattinger 1975; Kaplan and Yorke 1979). The dynamics of all these systems is generally chaotic and takes place on a single basin chaotic attractor, but is sometimes trapped near an unstable fixed point. When this happens, an orbit stays in the vicinity of the fixed point for an amount of time that depends on the distance from the fixed point and its dynamical properties. As a result, the system experiences a temporary suppression of chaos.

We propose to detect the existence of unstable fixed points in the mid-latitude atmospheric circulations by using recent results obtained for recurrences of dynamical systems (Freitas et al 2010; Lucarini et al 2012; Freitas et al 2012; Faranda et al 2013). These results have opened a new branch of research where recurrences of a certain observation in an orbit are treated via the statistics of extreme events. The novelty of this approach lies in the fact that classical extreme value laws can be found for such recurrences for almost every point of chaotic attractors (Freitas et al 2010). In (Faranda and Vaienti 2013) we have exploited this technique to study instrumental temperature records, and check that temperature recurrences obey one of the three classical extreme values, i.e. the atmosphere behaves as a chaotic system. Via this analysis, a map of European temperatures can be constructed whose recurrence is likely or unlikely within a certain time scale of interest.

In order to detect the possible unstable fixed points of the atmospheric dynamics, we will analyze several blocking indices. In general, a blocking index is defined in terms of the difference of pressure (or conjugated fields) between two different locations at the same longitude. When the flow is zonal, this difference always has the same sign because anticyclones are generally located at lower latitudes. Conversely, when the flow is blocked, low pressure systems tend to move to low latitudes and anticyclones to high latitudes, reversing the meridional gradient in pressure. A blocking event is identified as the persistence of such conditions for several days. 
We will begin our analysis with the Tibaldi-Molteni index (Tibaldi and Molteni 1990), defined at each longitude by differences of geopotential heights, and compare the results with those obtained for the bidimensional blocking index introduced by Pelly and Hoskins (2003), where differences are taken over a potential vorticity surface closer to the tropopause and to the core of the jet stream. After collecting evidence for the existence of unstable fixed points and their spatial distribution, we will perform the analysis on one-dimensional indices of atmospheric circulation to see whether they keep any trace of the existence of unstable fixed points. In particular, we will focus on the Artic Oscillation (AO) index, which is roughly a hemispheric average of the Tibaldi-Molteni index, and on the North Atlantic Oscillation (NAO) index, representative of the North-Atlantic/European regions only (NOAA 2015; Hurrell et al 2003).

The paper is organized as follows: in section 2, we give an overview of the method and explain the analogy between unstable fixed points and blocked circulation via dynamical systems toy models. In section 3, we present evidence for the existence of unstable fixed points by using the Tibaldi-Molteni and the Pelly blocking indices. In section 4, we analyze the role of one-dimensional indices, such as the AO and the NAO. Finally, we discuss how to improve the modeling of the mid-latitudes circulation on the basis of the results obtained.

\section{Method and results from dynamical systems}

In this section we A) show how to detect unstable fixed points in dynamical systems and time series, B) explain the inference procedures, and C) discuss the estimation of the parameters describing the statistical properties of the unstable point.

\subsection{Detection of unstable fixed points with dynamical systems techniques}

Let us consider a discrete-time dynamical system. This is a relevant hypothesis for an atmospheric system (Lucarini et al 2013), as both models and observations are made at discrete times. The dynamics is governed by the map $T$, which iterates the variables of the system $x$ according to

$$
x_{t+1}=T\left(x_{t}\right) .
$$

We assume that, by starting from a random initial condition, the dynamics follows a chaotic trajectory on a well-defined surface of the phase space, i.e. the attractor. We fix a point $\zeta$ on the attractor and measure the time series of the distances between $\zeta$ and the subsequent iterations of the orbit:

$$
w(t ; \zeta)=-\log \left(d\left(T\left(x_{t}\right), \zeta\right)\right),
$$

where $d$ is a distance function between two vectors. We are interested in the high extremes of $w(t ; \zeta)$, for all $t$. By construction, such extremes define the recurrences of the system. To identify the extremes, we apply the block-maxima approach. It consists of dividing the time series $T\left(x_{t}\right)$ into intervals of length $m$. Every $m$ observations, the closest recurrence to the point $\zeta$ is taken. If $n$ intervals of length $m$ are available in the series, one obtains $n$ closest recurrences. If the system is chaotic, the logarithmic weight forces the asymptotic extreme value distribution to follow a Gumbel law. A detailed explanation for this can be found in Faranda and Vaienti (2013), but the reason is intuitive: the Gumbel law is of the form 
$G(x)=\exp (-\exp (-x))$. One of the exponential functions comes from the exponential recurrence statistics, the other from the inverse of the logarithm. Other choices for $w$, typically power laws, constrain the asymptotic extreme distribution to be either a Weibull or a Fréchet distribution.

This theoretical framework applies to almost all the points of a chaotic attractor except at the unstable fixed points. A fixed point of the system in Eq. (1) is one that repeats itself under iteration, i.e. $T(x)=x$. An unstable or repelling fixed point is one for which the distance between itself and any point in a surrounding neighborhood increases under iterations (Katok and Hasselblatt 1997). A theoretical result from dynamical systems, obtained by Freitas et al (2012), states that when $\zeta$ is an unstable fixed point of the recurrence map $T$, then the distribution of $w(t ; \zeta)$ follows a modified Gumbel law:

$$
G(x, \theta)=\exp (-\exp (-\theta x)),
$$

where $\theta$ is a parameter known as the extremal index.

The concept of the extremal index originally appears in extreme value theory (Leadbetter et al 1983), where $\theta$ gives a measure of 'clustering', i.e. the tendency of a random process to exceed a threshold at consecutive times. If a threshold $u$ is applied to a series of observations $x_{1}, x_{2}, \ldots, x_{s}$, the exceedances are those for which $x_{i}>u$. Heuristically, the extremal index can then be thought of as $\theta=1 / \ell$, where $\ell$ is the mean duration of consecutive exceedances (clusters), i.e. the average of the time intervals spent above $u$.

In the dynamical systems context, $\theta$ can vary across the phase space depending on the $\zeta$ from which recurrences are measured (Freitas et al 2012). Clustering occurs when consecutive iterates of the orbit are observed near a point of the attractor. For almost all the nonsingular points there is no clustering. This means that, on average, an orbit enters the neighborhood of $\zeta$ once at time, which gives $\theta=1$. However, when $\zeta$ is close to an unstable fixed point, $\theta<1$. The smaller the $\theta$, the larger the cluster size, i.e. the longer the time the orbit stays in the vicinity of $\zeta$. In dynamical systems, the cluster extends in both time and space: we observe a time cluster in the minimum distances between the unstable fixed point and the orbit. This in turn effectively corresponds to a spatial cluster because the orbits are held within the vicinity of the unstable fixed point.

As an example, we consider the Hénon system (Hénon 1976) whose attractor is shown in Fig.1a, obtained by iterating the following set of equations:

$$
\begin{aligned}
& x_{t+1}=y_{t}+1-1.4 x_{t}^{2}, \\
& y_{t+1}=0.3 x_{t} .
\end{aligned}
$$

In this picture, the presence of the unstable fixed point $\zeta_{u}$ is not obvious. Its existence can be proved analytically by solving $T(x, y)=(x, y)$, with approximate solution $\zeta_{u}=(0.63,0.19)$, as indicated in Fig.1a. The dynamics around this point is different from that of a generic point, and this can be captured by computing the recurrences. Fig.1b and Fig.1c show distances of iterates of the orbit measured from $\zeta_{u}$ and a generic point $\zeta$ of the attractor. In the former case, a long cluster is visible and its length can be determined via the extremal index $\theta$, whose estimation we describe in the next section (for the Hénon map $\theta$ can also be computed analytically Freitas et al (2012)).

Although the Hénon dynamics is not representative of the atmospheric circulation, it is helpful to illustrate the general dynamical behavior around an unstable fixed point. In chaotic dynamical systems we can only observe clustering at unstable fixed points (see Freitas et al (2012), Theorem 1), independently 


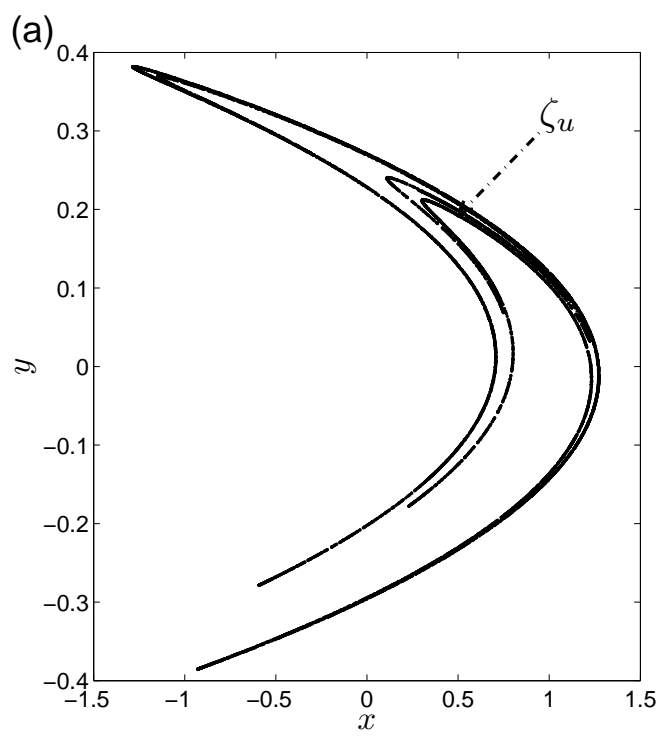

(b)

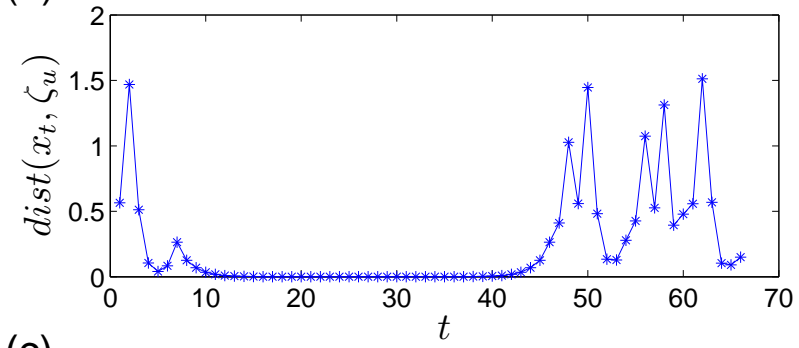

(c)

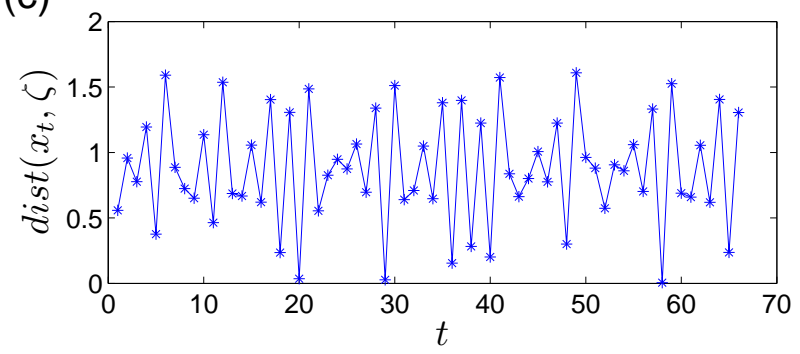

Fig. 1 a: Hénon attractor, obtained by iterating Eq. (2). The location of the unstable fixed point $\zeta_{u}$ is indicated. b: Series of distances from the unstable fixed point $\zeta_{u}$. Clustering occurs when the trajectory gets close to $\zeta_{u}$. c: Series of distances from a generic point of the attractor $\zeta$. No clustering occurs.

of the complexity of the system. If we consider the time series of observations as the output of a dynamical system, then we may hope to track the presence of unstable fixed points by measuring $\theta<1$ for some reference values $\zeta$.

2.2 Algorithm and inference of the extremal index

In order to compute the extremal index $\theta$ for time series we use the following algorithm:

1. Consider a time series consisting of $s$ observations: $\left\{x_{t}: t=1,2, \ldots, s\right\}$.

2. Fix $\zeta$ to be a point of the series itself.

3. Compute the series $w_{t}(\zeta)=-\log \left(d\left(x_{t}, \zeta\right)\right)$.

4. Take a very high quantile $q$ of the $w_{t}$ distribution, in order to consider only the closest recurrences (when $d(x, \zeta)$ is small, $w(x, \zeta)$ is large).

5 . Compute the extremal index.

A number of estimators exist for $\theta$. The simplest of these, the so-called "runs" or "blocks" methods, approximate $\theta$ by dividing the number of recurrent clusters by the total number of recurrences (Embrechts et al 1997). The cluster could be defined as a consecutive sequence of recurrent observations, terminating when an observation is no longer recurrent (by falling outside the small interval that defines recurrences). Depending on the context, different definitions of clusters may be appropriate. Another estimator, (Ferro and Segers 2003), is constructed out of the second moment of times between recurrences. For the analysis presented in this paper, we adopt another estimator by Süveges (2007), and we have checked that our results are robust across extremal index estimators. For fixed quantile $q$, Süveges' estimator reads: 


$$
\theta=\frac{\sum_{i}^{N_{c}}(1-q) S_{i}+N+N_{c}-\sqrt{\left(\sum_{i}^{N_{c}}(1-q) S_{i}+N+N_{c}\right)^{2}-8 N c \sum_{i}^{N_{c}}(1-q) S_{i}}}{2 \sum_{i}^{N_{c}}(1-q) S_{i}},
$$

where $N$ is the number of $w(t, \zeta)$ above the chosen quantile (i.e. recurrences), $N_{c}$ the number of observations which form a cluster of at least two consecutive recurrences, and $S_{i}$ the length of each cluster $i$. For details of the derivation of this estimator, see Süveges (2007).

\subsection{Finite-size effects}

Faranda et al (2011) discussed some of the problems related to the finiteness of datasets when studying the recurrences around a certain value in a time series. The results $\theta=1$ at generic points and $\theta<1$ at unstable fixed points hold only in the limit $q \rightarrow 1$, i.e. for the closest recurrences. When dealing with finite data sets, the limit $q \rightarrow 1$ is unattainable and, depending on the marginal distribution, some points $\zeta$ may be associated with an extremal index $\theta<1$ even when they are not unstable fixed points.

To illustrate this effect, we analyse a time series generated by an auto-regressive process of order 1 $(\mathrm{AR}(1)), x_{t}=\phi x_{t-1}+\sigma \epsilon_{t}$, where $|\phi|<1$ is the magnitude of the auto-regressive coefficient and $\epsilon_{t}$ is a random variable drawn from a normal distribution, $\sigma=0.1$ is a constant. It is known that the extremes of this process do not cluster in the limit $q \rightarrow 1$, so that $\theta=1$ for all $\zeta$ and all $\phi$. However, for finite datasets and fixed $q$, clustering will be observed among the weakly (exponentially) correlated exceedances all the while they have not exited an interval around $\zeta$ whose size is a function of $\phi$ and the underlying marginal density: for larger $\phi$ and $\zeta$ chosen in the wings of the marginal density, there is a greater 'finite-size' clustering.

In our numerical experiment, we take $\phi=0.5$ and synthetic datasets of lengths 30000 (similar to the real time series we later analyse) and 300000. We plot the empirical marginal densities in Fig. 2a , and the computed $\theta$ as a function of $\zeta$ in Fig. 2b. Note that $\theta<1$ in the wings of the distribution, even though the model has no inherent tendency to cluster extremes. Increasing the length of the datasets sharpens the estimate of the theoretical quantile of the marginal density. But it does not change the size of the interval that defines recurrences. For a fixed quantile (i.e. not increasing with sample size), the extremal index is therefore relatively insensitive to the sample size. For the AR(1) process it is possible to estimate $\theta$ analytically by calculating the mean sojourn time inside the recurrence interval. This time represents the length of a cluster — after which observables leave the interval, thereby terminating it. The inverse of the mean sojourn time thus provides an estimate of $\theta$. The analytical curve obtained is shown in Fig. 2b.

Our method to deal with such finite-size effects is based on the following observations: the extremal index obtained for some fixed $q<1$ depends on i) the shape of the marginal density, ii) the spectral properties of the process (i.e. $\phi$ in our above example), iii) the choice of $\zeta$, since clustering is a local property.

We have tested i) and ii) by changing both the marginal and the magnitude of the coefficient $\phi$, which is directly linked to the spectrum for the process (Brockwell and Davis 2002). We can go further by generating surrogates of the original dataset that have identical marginal distributions and, to within a very low tolerance, the same spectral properties. In this way we can 'subtract' the effects of i) and ii) in the computation of $\theta$. To perform this in practice, we use the Iterative Amplitude Adjusted Fourier Transform (IAAFT) of Schreiber and Schmitz (1996). In order to compute the residual extremal indices, 
which we will denote by $\theta^{*}(\zeta)$, it is sufficient to average over several surrogate estimates of $\theta$, such that:

$$
\theta^{*}(\zeta)=\left\langle\theta\left(\zeta, x_{\mathrm{SURR}}\right)\right\rangle-\theta(\zeta, x)
$$

where the \langle\rangle are averages over realizations of surrogate data. This procedure has been tested on the auto-regressive samples previously analysed. The residual $\theta^{*}$ is plotted in Fig. 2c and shows no local clustering.
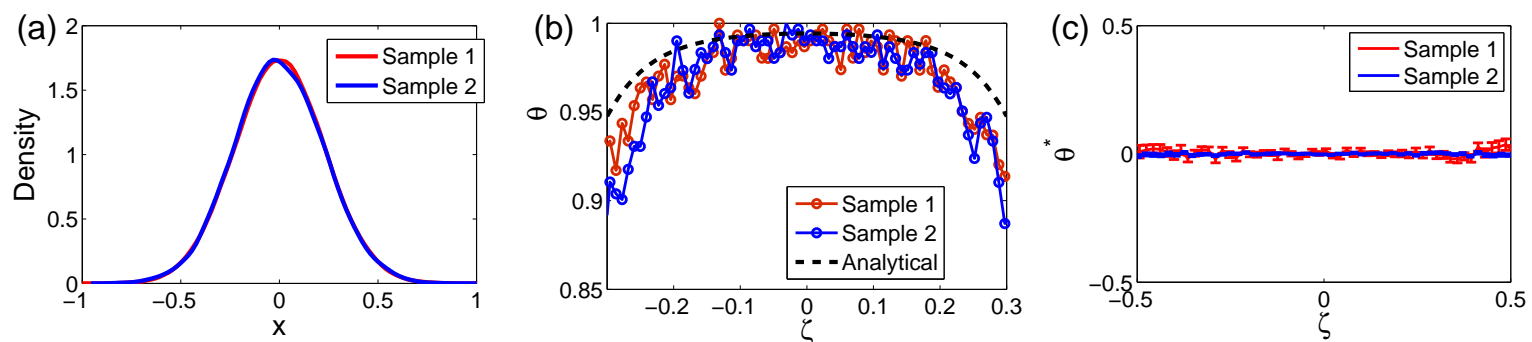

Fig. 2 a Empirical density for data generated by an autoregressive process. b: extremal indices $\theta$ computed at 100 reference points $\zeta$, together with its analytical estimate (dashed line). c: residual extremal index $\theta^{*}$ computed at the same $\zeta$ as in the central panel. Sample 1 contains 30000 data. Sample 2 contains 300000 data.

An analysis of the Hénon attractor provides a test of property iii). Here, we can check whether we are able to recover the known location of the unstable fixed point via a computation of $\theta^{*}$ for the time series of $x$ and $y$. Results are shown in Fig. 3 for 30000 observations. The location of $\zeta_{u}$ is picked out by the two peaks of $\theta^{*}$ at $x=0.63$ (Fig. 3a) and $y=0.19$ (Fig. 3b). Secondary peaks are also visible in these plots, which are related to the influence of $\zeta_{u}$ in nearby locations of the attractor.

(a)

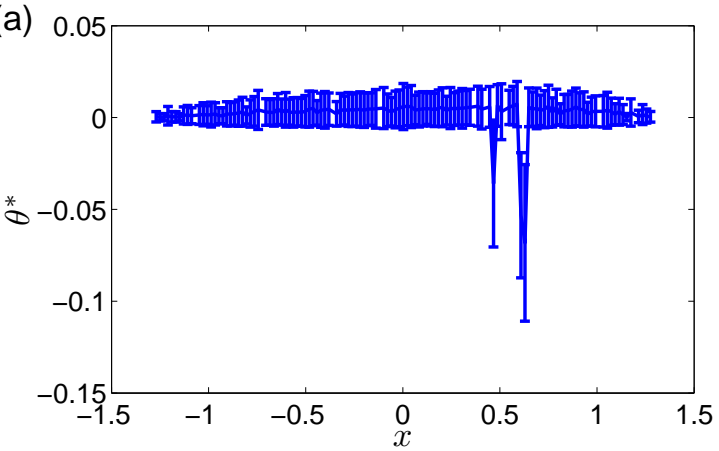

(b)

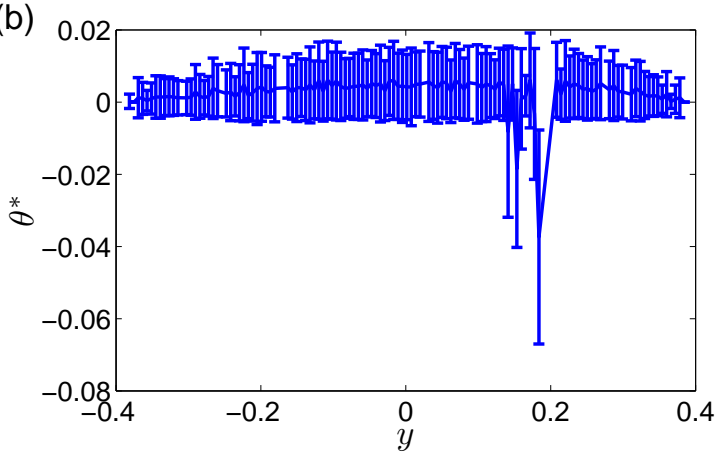

Fig. 3 Residual extremal index $\theta^{*}$, based on 30000 iterations of the Hénon map. a: $x$ observable. b: $y$ observable.

\section{Analysis of Multidimensional blocking indices}

The first bidimensional blocking index was introduced by Scherrer et al (2006) and it is based on the original definition given by Tibaldi and Molteni (1990); Tibaldi et al (1994). This index determines 
the longitudinal asymmetry of the atmospheric flow between $40^{\circ} \mathrm{N}$ and $80^{\circ} \mathrm{N}$, by comparing meridional gradients of geopotential height at $500 \mathrm{hPa}(Z)$. For each longitude in the northern extra-tropics, a southern gradient $B_{S}$ and a northern gradient $B_{N}$ of $Z$ are computed as follows:

$$
\begin{aligned}
& B_{S}=\frac{Z\left(\phi_{o}\right)-Z\left(\phi_{S}\right)}{\phi_{o}-\phi_{s}} \\
& B_{N}=\frac{Z\left(\phi_{n}\right)-Z\left(\phi_{0}\right)}{\phi_{n}-\phi_{s}}
\end{aligned}
$$

where $\phi_{n}=80^{\circ}+\delta, \phi_{0}=60^{\circ}+\delta, \phi_{s}=40^{\circ}+\delta, \delta=-5^{\circ}, 0^{\circ}, 5^{\circ}$. A given longitude is considered to be blocked at a given time if the following two conditions are satisfied for at least one value of $\delta$ :

$$
\text { (1): } B_{S}>0, \quad(2): B_{N}<-10 \mathrm{~m} / \text { degree }
$$

Here, we analyze daily time series of $B_{S}(t)$ computed for the $Z$ field of the National Centers for Environmental Prediction (NCEP) daily reanalysis (Kalnay et al 1996), which represent the strength of the Tibaldi-Molteni blocking index, under the condition that $B_{S}>0$ and $B_{N}<-10$. Blocking events mainly occur around $180^{\circ}$ (in the Pacific) and at $0^{\circ}$ (in the Eastern North Atlantic) longitude (d'Andrea et al 1998). For our analysis, positive values of $B_{S}(t)$ also satisfying condition $(2)$ are considered. The values of $\theta^{*}$ plotted in Fig. 4 are computed using the technique described in the previous section, with 100 reference points of $\zeta=B_{S}$. In the following analysis we generate 100 surrogates to compute $\left\langle\theta\left(\zeta, x_{\text {SURR }}\right)\right\rangle$, and take $q=0.995$. We find that our results are robust with respect to quantile provided $q \geq 0.99$.

(a)
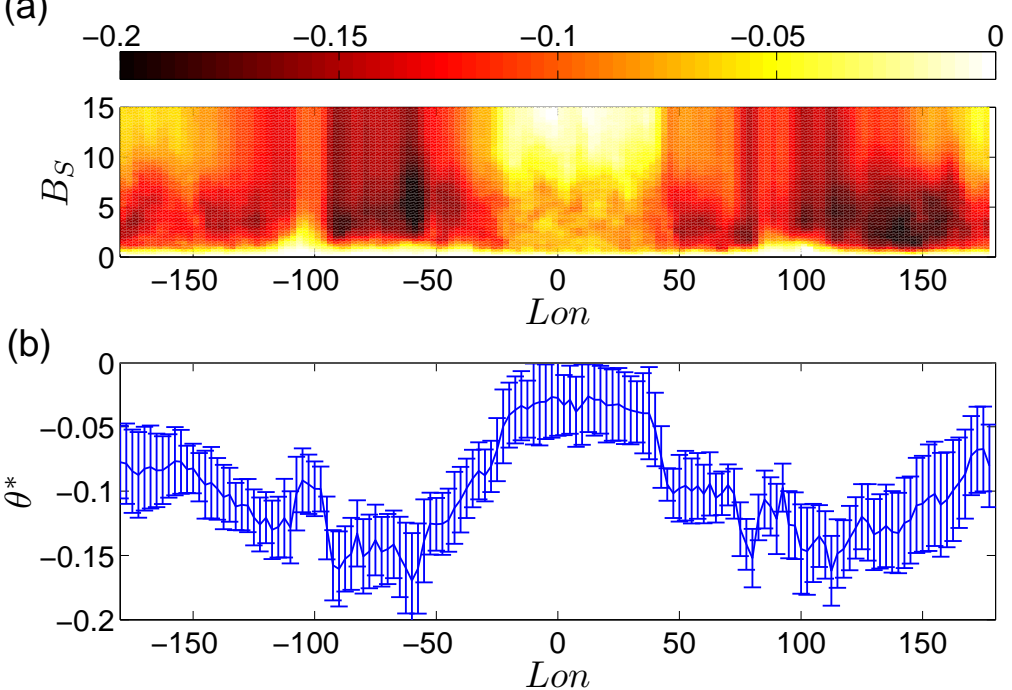

Fig. 4 a: Extremal indices $\theta^{*}$ (color scale) as a function of longitude Lon and the Tibaldi-Molteni blocking index $B_{S}(t)$. b: extremal index averaged over all $B_{S}$ vs longitude. Negative values indicate the presence of unstable fixed points. All the values are significant at a $95 \%$ level. 
Some areas attain negative values of $\theta^{*}$ and, over all, the average extremal index is negative at all longitudes (Fig. 4b). Therefore, for these areas the dynamics of blocking is compatible with the existence of unstable fixed points. It is quite surprising to observe that at $0^{\circ}$ longitude $\theta^{*}$ is close to zero (although the values of $\theta^{*}$ are significant also for this area, implying only moderate clustering. Although the European region is affected by strong blocking events, these area is not the one attaining the strongest clustering of $\left.B_{S}\right)$.

In order to have a detailed geographical description of the local clustering features, we will consider the local blocking index introduced by Pelly and Hoskins (2003) and Tyrlis and Hoskins (2008). This blocking index $B$ is a macroscopic measure of the strength of the meridional gradient of potential temperature on the isopotential vorticity surface with value 2 PVU (also called the PV2 surface; 1 PVU [ $1 \times 10^{-6} \mathrm{~K}$ $\mathrm{m}^{2} \mathrm{~kg}^{-1} \mathrm{~s}^{-1}$. This surface corresponds approximately to the tropopause, as described by Hoskins et al (1983). $B$ is computed every $5^{\circ}$ of longitude as the difference of the average of potential temperature for regions $15^{\circ}$ of latitude. Whenever $B$ takes positive values, it is referred to as local instantaneous blocking. Negative values of $B$ correspond instead to the westerlies mid-latitude circulation associated with the zonal flow. The more negative $B$, the stronger such a circulation.

We computed $\theta^{*}$ at each grid point for 100 values of $\zeta$, uniformly spaced between $\min (B)$ and $\max (B)$. We then averaged $\theta^{*}$ for three $B$ intervals corresponding respectively to blocked regimes $B>0$ (Fig. 5a) moderate zonal flows $-10<B<0$ (Fig. 5b), and strong zonal flow $B<-10$ (Fig. 5c). Only grid points with sufficient statistics to obtain reliable values of $\theta^{*}$ have been considered. For $B>0$ (blocked flows), $\theta^{*} \neq 0$ appears almost everywhere. Strong geographical differences appear in the distribution of $\theta^{*}$ : negative values are concentrated at higher latitudes, especially over Canada and central Asia where $\theta^{*} \simeq-0.15$. For the North-Atlantic sector, the dynamics over the Canadian region has been recognized by Vautard (1990) as a driver of the development of blocking structures. In the same paper, it has been argued that the precursor of transitions to the blocked circulation can be found by considering the weather evolution over this region. It is worth noticing that the unstable fixed points tend to be located in proximity or just downstream of the two major mountain chains (i.e. the Rocky Mountains and the Tibetan Plateau). Not surprisingly, these are a necessary ingredient to foster stationary waves (Brayshaw et al 2008). In low dimensional dynamical systems only the neighborhood of unstable fixed points is affected by clustering. this is not necessarily the case for spatially extended systems: the effect of the unstable fixed points can be transported in space by the dynamics. Unstable fixed points found with our analysis may be the preferred breaking points for the amplification of Rossby waves, or for the development of non-linear interactions. At a mathematical level, there are indeed evidence of the relation between unstable fixed points and the destabilization of systems governed by partial differential equations (see for example the work by Memory (1991) Example 2 for the delayed logistic equation with diffusion and reference therein). Evidences that this behavior is consistent with the atmospheric dynamics and the blocking phenomena can be found in (Christensen and Wiin-Nielsen 1996).

For the North Atlantic, the location of unstable fixed points is consistent with the one obtained by (Buehler et al 2011) -Fig 1a), where the blocking frequency is computed with a dynamical technique called the Lagrangian method which consists in incorporating in the blocking definition the contribution of cyclonic activity to reduce artificial maxima. The use of the surrogate acts similarly to this additional condition by removing the mean dynamical component, this might explains why the longitudinal profiles are different with respect to the ones obtained by Tibaldi and Molteni (1990) and other authors.

Fig. 5-b and c show the values of $\theta *$ respectively when $-10<B<0$ and $B<-10$. The more zonal the flow, the more negative $B$ and the less negative values of $\theta *$ are observed. This is compatible with the 
idea that unstable fixed points are associated to the blocked flow, as it will be confirmed by the analysis of the $\mathrm{AO}$ index is the next section.

The meridional averages of the $B>0$ are reported in Fig. 6. As for the Tibaldi-Molteni index we observe a complex dependence on both the longitude and the values of $B$. Almost everywhere the averages are negative and significant at a $95 \%$ level. It is important to compare the $\theta^{*}$ for $B$ with the ones obtained for the Tibaldi-Molteni index. We observe that the scaling is different: although significant, the values of $\theta^{*}$ for $B$ are almost 4 times smaller in magnitude than the value obtained for $B_{S}$. We conjecture that the Pelly index has smaller $\theta^{*}$ because it is computed by averaging over 15 degrees of latitude whereas the Tibaldi Molteni over 50 degrees of latitude (35N to $85 \mathrm{~N})$. If we plot together the results for the two indexes by rescaling them with the factor $10 / 3$ (equivalent to the latitude ratio), we obtain good agreement as shown in Fig. 7. Tests performed on low dimensional lattice systems indicate that the averaging window have an impact on the magnitude of $\theta^{*}$ but this will need to be further investigated to get a complete understanding of the results. For the moment, we just note that by doing such a rescaling, results are consistent over a wide range of longitudes except the Asian area.

(a)

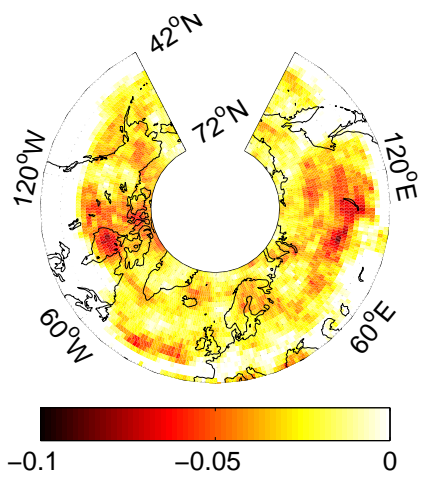

(b)

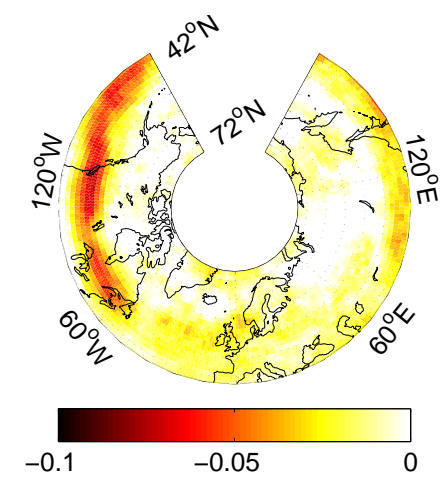

(c)

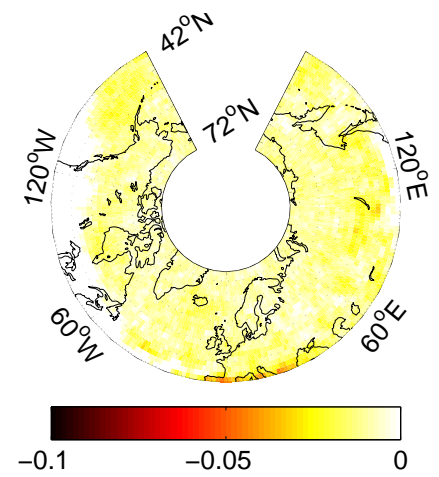

Fig. 5 Residual extremal index $\theta^{*}$ for three different ranges of the bidimensional blocking index $B$ defined by Pelly et al. Pelly and Hoskins (2003). a: $\theta^{*}$ averaged over $B>0$ values corresponding to blocked flow regimes. b: $\theta^{*}$ averaged over $-10<B<0$ values corresponding to weak zonal flow regime. c: $\theta^{*}$ averaged over $B<-10$ values corresponding to strong zonal flow regime. See text for descriptions. 
(a)
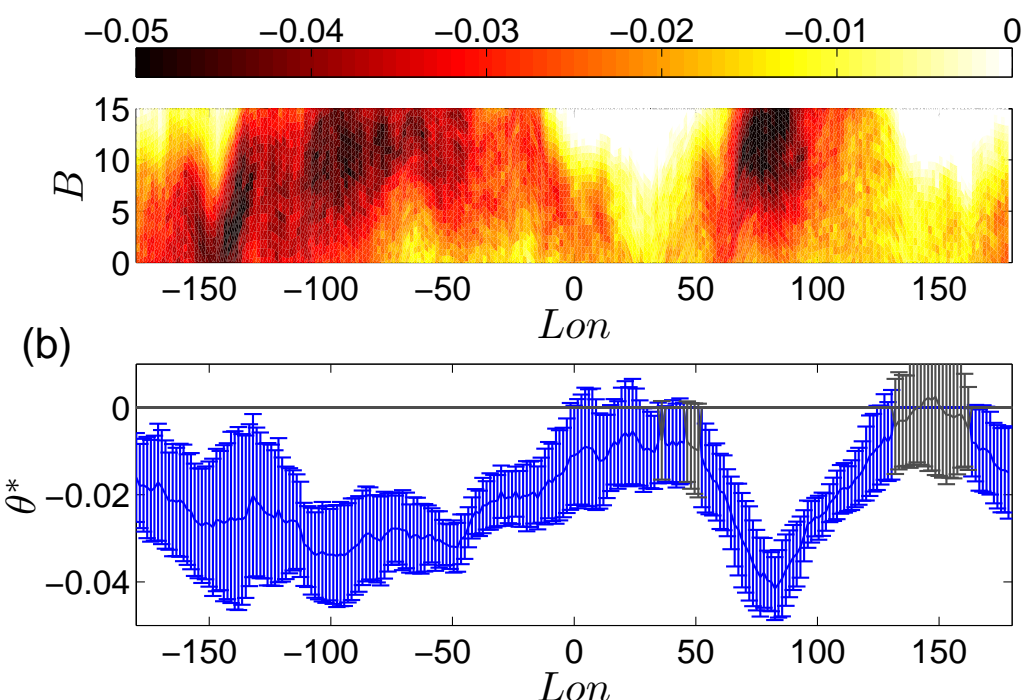

Fig. 6 a: Residual Extremal indices $\theta^{*}$ versus longitude for the blocking index $B$. Negative values indicate the presence of unstable fixed points. b: Meridional average of $\theta^{*}$ between $35 \mathrm{~N}$ and $70 \mathrm{~N}$. The blue errorbars show the significant results at the $95 \%$ level and represent the standard deviation of the mean over different latitudes.

\section{Analysis of NAO and AO Atmospheric indices}

Although local indices provide comprehensive geographical information about the atmospheric circulation, one-dimensional indicators have been extensively used to characterize and forecast specific phenomena (see Hurrell and Deser (2010) and references therein).

Over Europe, the transition between zonal and blocked atmospheric dynamics has been historically associated to the North Atlantic Oscillation (NAO) index, originally defined as the difference in pressure between Lisbon and Reykjavik (Hurrell 1995). Here we use the NOAA version of such index available via (NOAA 2015) computed by using the Rotated Principal Component Analysis (RPCA) used by Barnston and Livezey (1987). The positive phase of the NAO reflects below-normal heights and pressure across the high latitudes of the North Atlantic and above-normal heights and pressure over the central North Atlantic, the eastern United States and western Europe. The negative phase reflects an opposite pattern of height and pressure anomalies over these regions (Hurrell et al 2003).

The Arctic Oscillation index $(\mathrm{AO})$ is more representative of the blocking dynamics over the entire northern hemisphere: it is constructed by projecting the daily 0 UTC $1000 \mathrm{mb}$ height anomalies pole-ward of $20^{\circ} \mathrm{N}$ onto the leading mode of the Empirical Orthogonal Function (EOF) analysis of monthly mean 1000mb height during the years 1950-2014 (Thompson and Wallace 1998). Hence, the AO index behaves like a zonal average of the Tibaldi-Molteni index. In the negative phase, the polar low pressure system (also known as the polar vortex) over the Arctic is weaker, which results in weaker zonal flow. When the $\mathrm{AO}$ is positive the polar circulation is stronger and forces cold air and storms to remain farther north. The NAO and AO indices exhibit considerable inter-seasonal and inter-annual variability, and prolonged 


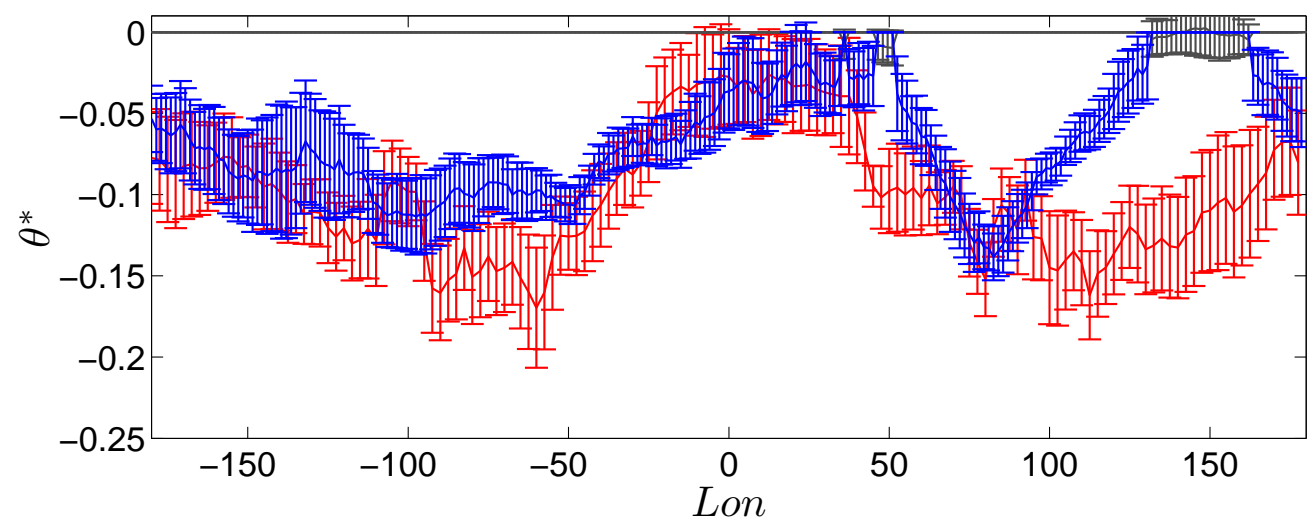

Fig. 7 Comparison between $\theta^{*}$ obtained for the Tibaldi-Molteni index (red errorbars) and $\frac{10}{3} \theta^{*}$ for the $B$ index (blue errorbars for significant values at $95 \%$ and grey non significant values). The errobars for the $B$ index have been multiplied by a factor $\sqrt{10 / 3}$. The factor $10 / 3$ is the ratio between the area considered to compute the Tibaldi Molteni index with respect to the $B$ index.

periods of both positive and negative phases of the pattern are not rare (Thompson and Wallace 1998). The daily NAO and AO data used in this paper are maintained by the US Climate Prediction Center an can be downloaded via the NOAA webiste (NOAA 2015).

With the analysis of a one-dimensional index, we can explore the link between complex atmospheric dynamics and simple one-dimensional observables. A priori, there is no reason why the two indices should provide the same information. The $\mathrm{AO}$ is a hemispheric average, the $\mathrm{NAO}$ a regional index. Even though they are both one-dimensional time series, we will keep in mind their different origin.

We start by analysing the time series and the histograms shown in Fig. 8. The distributions of both $\mathrm{NAO}$ and AO are unimodal and peaked around zero, roughly similar to a Gaussian. The indices seem to spend most of their time around zero values with noisy fluctuations superimposed. A thirty-day moving average filter (green curves) reproduces a unimodal histogram, and there is no evidence that the time series oscillates between two states. It is therefore hard to recognize in the data any trace of bistability or multistability.

Even if we look at single episodes, a dynamical structure compatible with the existence of an unstable fixed point remains unclear: let us consider two examples of negative NAO and AO phases (Fig. 9a-d) and one positive phase (Fig. 9e and Fig. 9f) recorded respectively for September 2002, December 2010 and January 1988. For the 2002, the negative phases of NAO and AO seem to be comparable with the dynamics of the Hénon attractor around the fixed point $\zeta_{u}$ (Fig. 1b)), but the duration and intensity of the negative phases are different. In December 2010, Europe experienced a severe cold spell. Although the $\mathrm{NAO}$ and the $\mathrm{AO}$ indices settle to negative values, the $\mathrm{NAO}$ oscillates over several values resembling that of a chaotic variable, whereas the $\mathrm{AO}$ seems to cluster for consecutive days around values of -4 and -2 . For January 1988, the behavior of the indices look much more chaotic with oscillations associated with the mean lifetime of baroclinic structures (a few days). This latter regime can be compared to Fig.1c), 
(a)

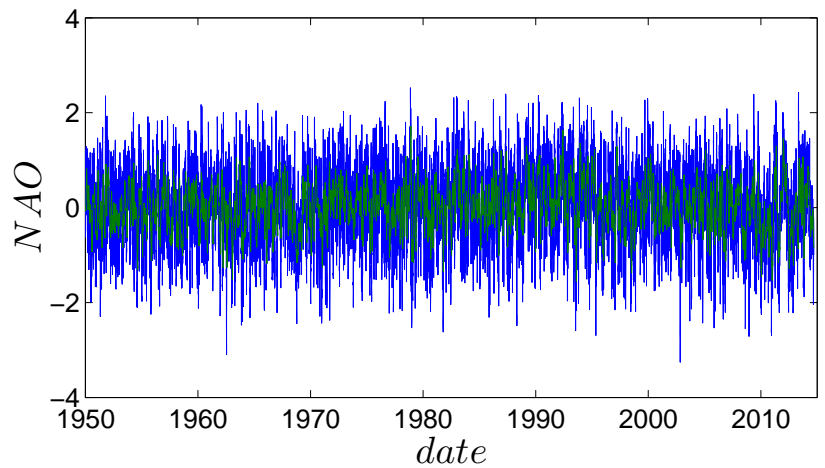

(c)

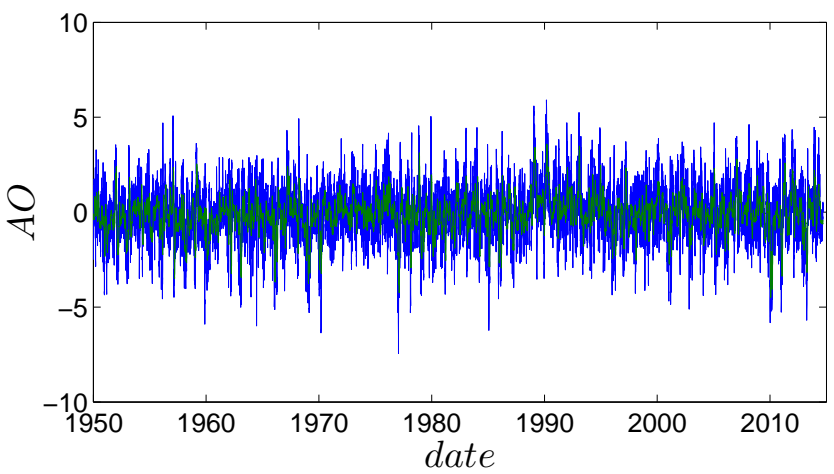

(b)

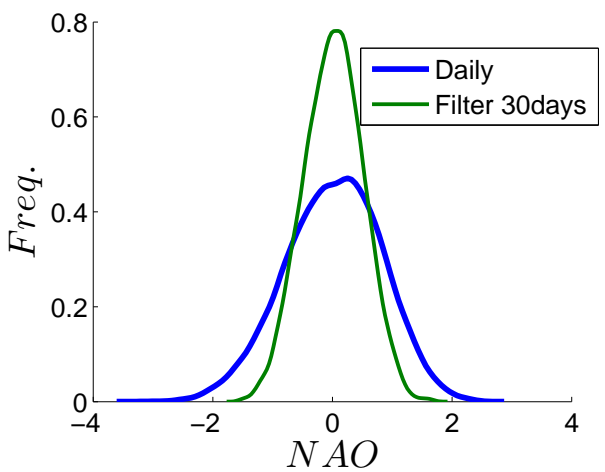

(d)

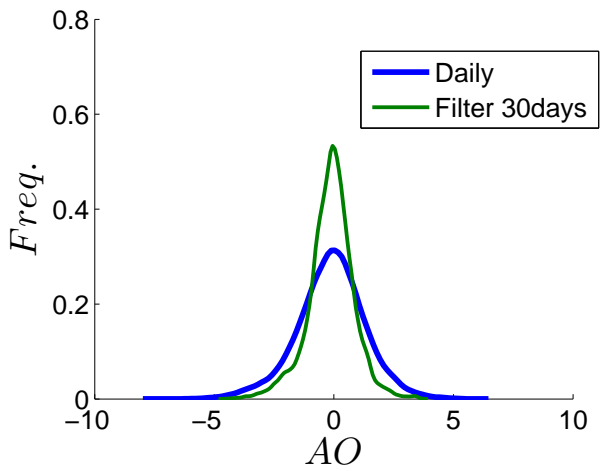

Fig. 8 NAO (a) and AO (c) daily time series and their empirical density functions (b and $\mathbf{d}$ respectively ). Blue: original dataset, green: moving average filter over a 30 day window.

i.e. with a typical point of the Hénon attractor. The contradicting results obtained for single episodes imply that the existence of an unstable fixed point must be assessed statistically via the computation of $\theta^{*}$.

After applying the procedure described in Section 2-B, we obtain, for each value of the NAO and AO, the residual extreme value index $\theta^{*}$, as shown in Fig. 10. We recall that for NAO and AO values around zero there is no additional clustering, i.e. no unstable fixed points can be detected. The behavior of the two indices is indeed different. $\theta^{*}$ is negative for negative $\mathrm{AO}$, following the core hypothesis of this paper that blocked circulation can be associated with the existence of unstable fixed points. In contrast, $\theta^{*}$ is positive for positive NAO.

The relation between negative $\theta^{*}$ and blocking for the $\mathrm{AO}$ can be explained if we look at the average sea level pressure fields for the days such that $\mathrm{AO}<-4$, i.e. $\theta^{*} \simeq-0.15$. This average is shown in Fig. $11 \mathrm{a}$ is compared against the average over the remaining days represented in Fig. 11b. They reproduce respectively the blocked flow with an high over Iceland and a low pressure systems over the Azore Islands, and the usual zonal flow with the Azore anticyclone and a low pressure located between Greenland and Iceland. 
(a)

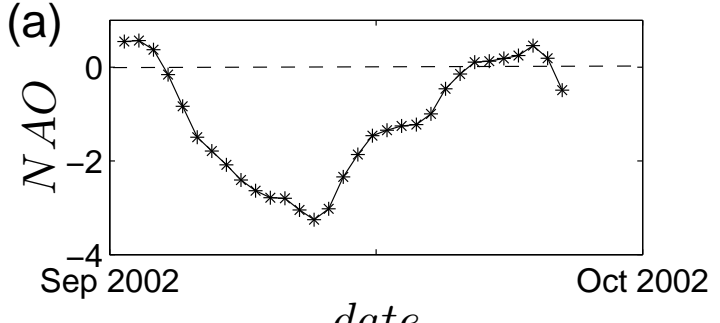

(c)

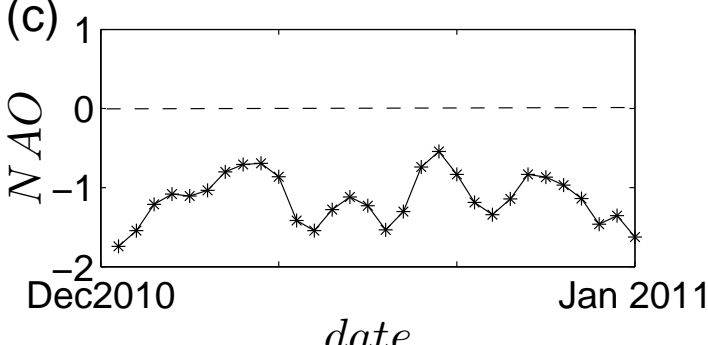

(e)

date

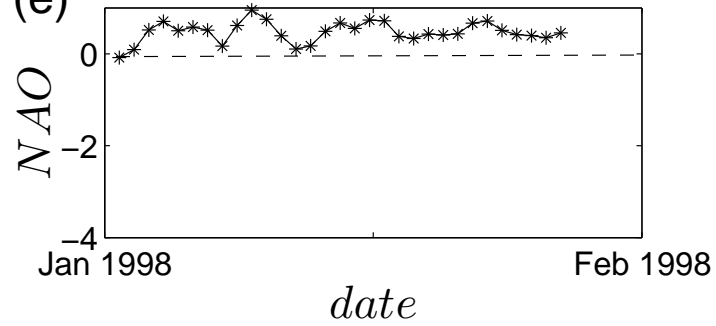

(b) 2

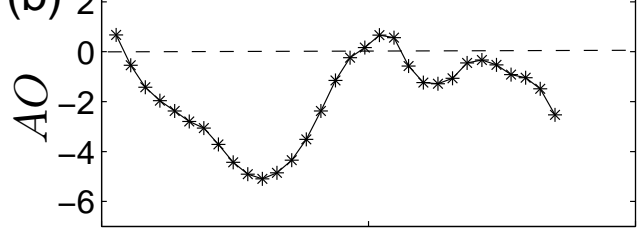

Sep 2002
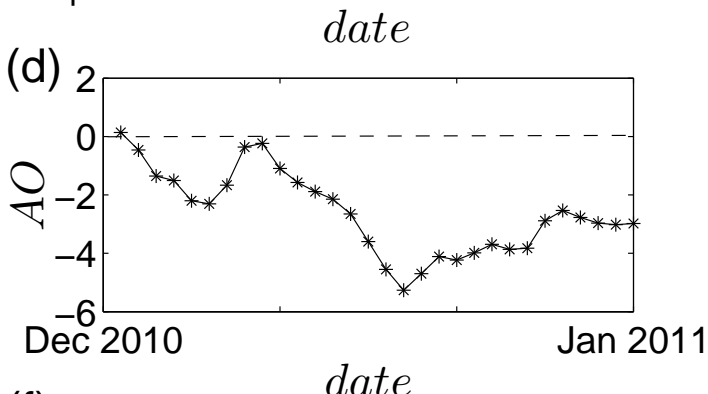

(f)

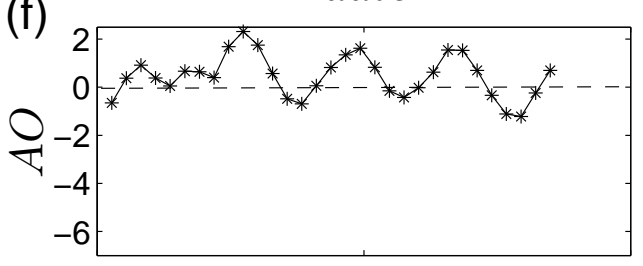

Jan 1988

date

Fig. 9 NAO (a-c-e) and AO (b-c-d) daily time series for some specific events. a-b: September 2002. c-d: December 2010. e-f: January 1988

A priori, there is no reason why the two indices should follow the same behavior. Features of the NAO are compatible with that observed for the Tibaldi-Molteni and the Pelly index around $0^{\circ}$ longitude, where the blocked flow was not associated with negative $\theta^{*}$. The disruption of clusters for positive NAO corresponding to zonal flow is compatible with the presence of complex geography, which tends to destroy the typical time scales of baroclinic instability (1.5 to 3 days). It is encouraging to find the trace of the existence of unstable fixed points for the AO index, i.e., that the hemispheric average does not erase the clustering properties found for $B$ and $B_{S}$. This analysis indeed suggests that the $A O$ is more sensitive to blocking phenomena than the $N A O$. We thus can account for the empirical observation of (Ambaum et al 2001).

\section{Conclusion and Discussion}

In this paper, we have adapted the concept of the extremal index, as applied in dynamical systems, to the analysis of atmospheric indices which describe the switching between zonal flow to blocked flow in the atmospheric mid-latitude circulation. We have presented evidence that the switching between atmospheric and blocked circulation can be associated with the existence of an unstable (saddle) fixed point 

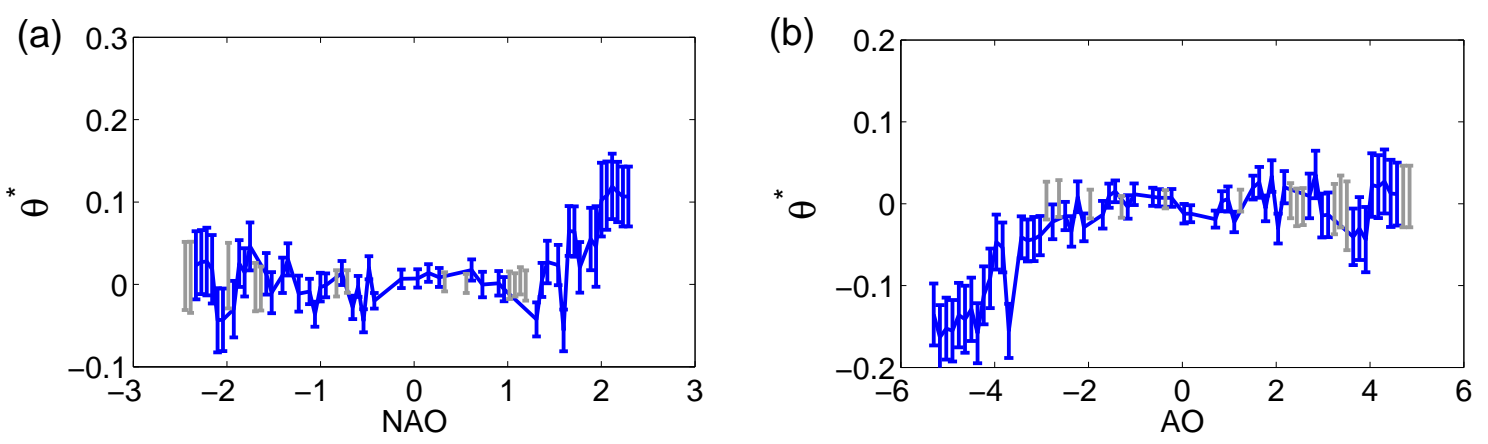

Fig. 10 Residual extremal index $\theta^{*}$ for the NAO index (a) and the AO index (b). Error bars represent a standard deviation of the mean taken over the ensemble of 100 surrogates. Blue: significant values at $95 \%$ level. Grey: non significant values

(a)

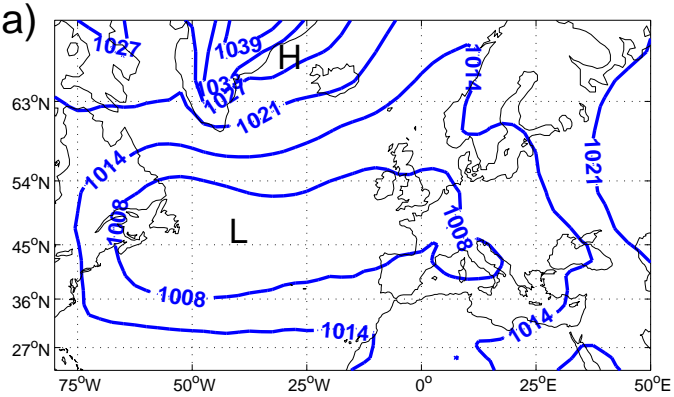

(b)

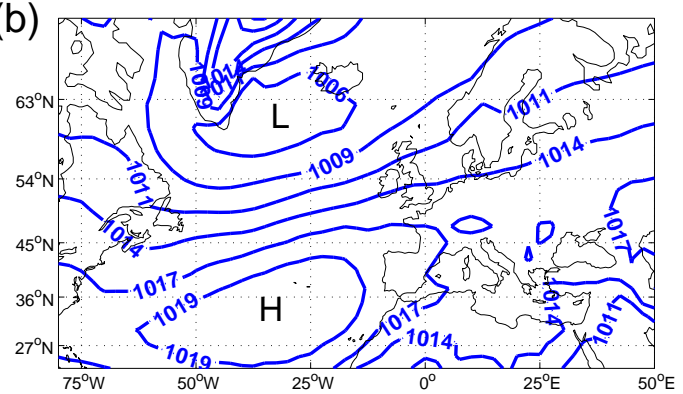

Fig. 11 a: Sea level pressure field averaged over all the days corresponding to the presence of unstable fixed point $(\mathrm{AO}<-4)$. b: sea level pressure field averaged over all remaining days.

of the atmospheric dynamics. The novelty of this approach lies in the use of observations, rather than intermediate complexity models or GCM. Our results appear to be robust across blocking indices, and are consistent with mid-latitude circulation mechanisms and local geography.

Such information is preserved in the AO time series, which is a sort of hemispheric average of the TibaldiMolteni index. On the other hand, it shows that the NAO index does not keep track of the presence of unstable fixed point. This result seems to contradict the fact that blocking events can be often be tracked by a negative phase of the NAO index (Hurrell et al 2003). However, we remark that the operative definition of blocking used in forecasts centers such as the NOAA strongly differs from the dynamical one employed for the computation of unstable fixed points: the first definition requires both large negative values of the NAO and the persistence for 5 days, the dynamical one requires the persistence of the same negative values for several days, independently on its magnitude. Moreover, as detailed in (Memory 1991), when partial differential equations govern the dynamics of a system, the presence of an unstable fixed point may trigger instabilities which propagate in space, thus creating long range interactions (teleconnections) between the initial location of unstable fixed point and the region affected. Our analysis seems to point to one of the mechanisms invoking non-linear interactions, either between zonal flow and eddies or between planetary waves (Charney and DeVore 1979; Egger 1978; Kung et al 1990; Christensen 
and Wiin-Nielsen 1996) as the driving mechanisms for the blocking phenomena. The complex spatial distribution of unstable fixed points let us discard the possibility that simple bi-stability mechanisms could explain the transitions between blocking and zonal flow, as proposed in (Tung and Lindzen 1979; Shutts 1983, 1986).

This paper also suggests a novel approach to statistical and dynamical modeling: in Masato et al (2009) the properties of blocking indices have been widely investigated and compared to the statistics of stationary red noise process. The authors argued that the statistical model was not sufficient to describe the characteristics of blocking and claimed that the persistence beyond that given by a red noise model is due to the self-sustaining nature of the blocking phenomenon. Here, we have shed light on this self-sustaining nature: When the circulation settles in a blocked regime, the presence of unstable fixed points gives rise to the persistence (the self-sustaining nature) of quasi-stationary conditions. In order to improve the statistical modeling of blocking phenomena, one has to account for local clustering effects in statistical models, which is a non-trivial challenge.

\section{Acknowledgements}

DF and PY were supported by the ERC Grant A2C2 (No. 338965). We thanks two anonymous referees whose suggestions greatly improved the quality of this paper. DF thanks M.Carmen Alvarez-Castro for useful discussions.

\section{References}

Ambaum MH, Hoskins BJ, Stephenson DB (2001) Arctic oscillation or north atlantic oscillation? Journal of Climate 14(16):3495-3507

Barnston AG, Livezey RE (1987) Classification, seasonality and persistence of low-frequency atmospheric circulation patterns. Monthly weather review 115(6):1083-1126

Barriopedro D, García-Herrera R, Lupo AR, Hernández E (2006) A climatology of northern hemisphere blocking. Journal of Climate 19(6):1042-1063

Barriopedro D, García-Herrera R, Trigo R (2010) Application of blocking diagnosis methods to general circulation models. part i: A novel detection scheme. Climate dynamics 35(7-8):1373-1391

Barriopedro D, Fischer EM, Luterbacher J, Trigo RM, García-Herrera R (2011) The hot summer of 2010: redrawing the temperature record map of europe. Science 332(6026):220-224

Beniston M (2004) The 2003 heat wave in europe: A shape of things to come? an analysis based on swiss climatological data and model simulations. Geophysical Research Letters 31(2)

Benzi R, Paladin G, Parisi G, Vulpiani A (1985) Characterisation of intermittency in chaotic systems. Journal of Physics A: Mathematical and General 18(12):2157

Brayshaw DJ, Hoskins B, Blackburn M (2008) The storm-track response to idealized sst perturbations in an aquaplanet gcm. Journal of the Atmospheric Sciences 65(9):2842-2860

Brockwell PJ, Davis RA (2002) Introduction to time series and forecasting, vol 1. Taylor \& Francis

Buehler T, Raible CC, Stocker TF (2011) The relationship of winter season north atlantic blocking frequencies to extreme cold or dry spells in the era-40. Tellus A 63(2):212-222

Charney JG (1947) The dynamics of long waves in a baroclinic westerly current. Journal of Meteorology 4(5):136-162

Charney JG, DeVore JG (1979) Multiple flow equilibria in the atmosphere and blocking. Journal of the atmospheric sciences 36(7):1205-1216

Christensen C, Wiin-Nielsen A (1996) Blocking as a wave-wave interaction. Tellus A 48(2):254-271

Colucci SJ, Alberta TL (1996) Planetary-scale climatology of explosive cyclogenesis and blocking. Monthly weather review 124(11):2509-2520

d'Andrea F, Tibaldi S, Blackburn M, Boer G, Déqué M, Dix M, Dugas B, Ferranti L, Iwasaki T, Kitoh A, et al (1998) Northern hemisphere atmospheric blocking as simulated by 15 atmospheric general circulation models in the period 1979-1988. Climate Dynamics 14(6):385-407 
Dole R, Hoerling M, Perlwitz J, Eischeid J, Pegion P, Zhang T, Quan XW, Xu T, Murray D (2011) Was there a basis for anticipating the 2010 russian heat wave? Geophysical Research Letters 38(6)

Dole RM (1986) Persistent anomalies of the extratropical northern hemisphere wintertime circulation: Structure. Monthly weather review 114(1):178-207

Egger J (1978) Dynamics of blocking highs. Journal of the Atmospheric Sciences 35(10):1788-1801

Emanuel K (2005) Increasing destructiveness of tropical cyclones over the past 30 years. Nature 436(7051):686-688

Embrechts P, Klüppelberg C, Mikosch T (1997) Modelling Extremal Events for Insurance and Finance. Springer-Verlag, Berlin Heidelberg

Faranda D, Vaienti S (2013) A recurrence-based technique for detecting genuine extremes in instrumental temperature records. Geophysical Research Letters 40(21):5782-5786

Faranda D, Lucarini V, Turchetti G, Vaienti S (2011) Numerical convergence of the block-maxima approach to the generalized extreme value distribution. Journal of Statistical Physics 145(5):1156-1180

Faranda D, Freitas JM, Lucarini V, Turchetti G, Vaienti S (2013) Extreme value statistics for dynamical systems with noise. Nonlinearity 26(9):2597

Ferro CAT, Segers J (2003) Inference for clusters of extreme values. J R Statist Soc B pp 515-528

Frederiksen J (1982) A unified three-dimensional instability theory of the onset of blocking and cyclogenesis. Journal of the Atmospheric Sciences 39(5):969-982

Freitas ACM, Freitas JM, Todd M (2010) Hitting time statistics and extreme value theory. Probability Theory and Related Fields 147(3-4):675-710

Freitas ACM, Freitas JM, Todd M (2012) The extremal index, hitting time statistics and periodicity. Advances in Mathematics 231(5):2626-2665

Ghil M (1987) Dynamics, statistics and predictability of planetary flow regimes. In: Irreversible Phenomena and Dynamical Systems Analysis in Geosciences, Springer, pp 241-283

Hansen AR (1986) Observational characteristics of atmospheric planetary waves with bimodal amplitude distributions. Advances in Geophysics 29:101-133

Hénon M (1976) A two-dimensional mapping with a strange attractor. Communications in Mathematical Physics 50(1):6977

Holton JR, Hakim GJ (2013) An introduction to dynamic meteorology. Academic press

Hoskins BJ, James IN, White GH (1983) The shape, propagation and mean-flow interaction of large-scale weather systems. Journal of the atmospheric sciences 40(7):1595-1612

Hurrell JW (1995) Decadal trends in the north atlantic oscillation: regional temperatures and precipitation. Science 269(5224):676-679

Hurrell JW, Deser C (2010) North atlantic climate variability: the role of the north atlantic oscillation. Journal of Marine Systems $79(3): 231-244$

Hurrell JW, Kushnir Y, Ottersen G, Visbeck M (2003) An overview of the North Atlantic oscillation. Wiley Online Library

Kalnay E, Kanamitsu M, Kistler R, Collins W, Deaven D, Gandin L, Iredell M, Saha S, White G, Woollen J, et al (1996) The ncep/ncar 40-year reanalysis project. Bulletin of the American meteorological Society 77(3):437-471

Kaplan JL, Yorke JA (1979) Chaotic behavior of multidimensional difference equations. In: Functional Differential equations and approximation of fixed points, Springer, pp 204-227

Katok A, Hasselblatt B (1997) Introduction to the modern theory of dynamical systems, vol 54. Cambridge university press

Kung EC, Dacamara CC, Baker WE, Susskind J, Park CK (1990) Simulations of winter blocking episodes using observed sea surface temperatures. Quarterly Journal of the Royal Meteorological Society 116(495):1053-1070

Leadbetter MR, Lindgren G, Rootzén H (1983) Extremes and Related Properties of Random Sequences and Processes. Springer-Verlag, New York, Heidelberg, Berlin

Legras B, Ghil M (1985) Persistent anomalies, blocking and variations in atmospheric predictability. Journal of the atmospheric sciences $42(5): 433-471$

Lucarini V, Faranda D, Turchetti G, Vaienti S (2012) Extreme value theory for singular measures. Chaos: An Interdisciplinary Journal of Nonlinear Science 22(2):023,135

Lucarini V, Blender R, Herbert C, Pascale S, Wouters J (2013) Mathematical and physical ideas for climate science. arXiv preprint arXiv: 13111190

Lupo AR, Smith PJ (1995) Climatological features of blocking anticyclones in the northern hemisphere. Tellus A 47(4):439456

Masato G, Hoskins BJ, Woollings TJ (2009) Can the frequency of blocking be described by a red noise process? Journal of the Atmospheric Sciences 66(7):2143-2149

Masato G, Hoskins B, Woollings TJ (2012) Wave-breaking characteristics of midlatitude blocking. Quarterly Journal of the Royal Meteorological Society 138(666):1285-1296

McWilliams JC, Flierl GR, Larichev VD, Reznik GM (1981) Numerical studies of barotropic modons. Dynamics of Atmospheres and Oceans 5(4):219-238 
Memory MC (1991) Stable and unstable manifolds for partial functional differential equations. Nonlinear Analysis: Theory, Methods \& Applications 16(2):131-142

Mo K, Ghil M (1988) Cluster analysis of multiple planetary flow regimes. Journal of Geophysical Research: Atmospheres (1984-2012) 93(D9):10,927-10,952

Nakamura H, Nakamura M, Anderson JL (1997) The role of high-and low-frequency dynamics in blocking formation. Monthly Weather Review 125(9):2074-2093

NOAA (2015) North atlantic oscillation. URL http://www.cpc.ncep.noaa.gov/data/teledoc/nao.shtml

Payne LE, Sattinger D (1975) Saddle points and instability of nonlinear hyperbolic equations. Israel Journal of Mathematics $22(3-4): 273-303$

Pelly JL, Hoskins BJ (2003) A new perspective on blocking. Journal of the atmospheric sciences 60(5):743-755

Rudeva I (2008) On the relation of the number of extratropical cyclones to their sizes. Izvestiya, Atmospheric and Oceanic Physics 44(3):273-278

Schär C, Jendritzky G (2004) Climate change: hot news from summer 2003. Nature 432(7017):559-560

Scherrer SC, Croci-Maspoli M, Schwierz C, Appenzeller C (2006) Two-dimensional indices of atmospheric blocking and their statistical relationship with winter climate patterns in the euro-atlantic region. International journal of climatology 26(2):233-249

Schreiber T, Schmitz A (1996) Improved surrogate data for nonlinearity tests. Physical Review Letters 77(4):635

Shutts G (1983) The propagation of eddies in diffluent jetstreams: Eddy vorticity forcing of blockingflow fields. Quarterly Journal of the Royal Meteorological Society 109(462):737-761

Shutts G (1986) A case study of eddy forcing during an atlantic blocking episode. Advances in Geophysics 29:135-162

Simmons A, Wallace J, Branstator G (1983) Barotropic wave propagation and instability, and atmospheric teleconnection patterns. Journal of the Atmospheric Sciences 40(6):1363-1392

Süveges M (2007) Likelihood estimation of the extremal index. Extremes 10(1-2):41-55

Thompson DW, Wallace JM (1998) The arctic oscillation signature in the wintertime geopotential height and temperature fields. Geophysical Research Letters 25(9):1297-1300

Tibaldi S, Molteni F (1990) On the operational predictability of blocking. Tellus A 42(3):343-365

Tibaldi S, Tosi E, Navarra A, Pedulli L (1994) Northern and southern hemisphere seasonal variability of blocking frequency and predictability. Monthly Weather Review 122(9):1971-2003

Tung K, Lindzen R (1979) A theory of stationary long waves. i-a simple theory of blocking. ii-resonant rossby waves in the presence of realistic vertical shears

Tyrlis E, Hoskins B (2008) Aspects of a northern hemisphere atmospheric blocking climatology. Journal of the Atmospheric Sciences 65(5):1638-1652

Vautard R (1990) Multiple weather regimes over the north atlantic: Analysis of precursors and successors. Monthly Weather Review 118(10):2056-2081

Wallace J, Blackmon M (1983) Observations of low-frequency atmospheric variability. Large-scale dynamical processes in the atmosphere(A 84-15488 04-47) London, Academic Press, 1983, pp 55-94

Weeks ER, Crocker JC, Levitt AC, Schofield A, Weitz DA (2000) Three-dimensional direct imaging of structural relaxation near the colloidal glass transition. Science 287(5453):627-631 